Supporting information for

\title{
Unveiling The Relationship Between Energy Transfer and Triplet Energy Level by Tuning Diarylethene within Eu(III) \\ Complexes
}

Zhixiang Zhang, ${ }^{\ddagger}$ Lingjun He, ${ }^{\ddagger}$ Jing Feng,,${ }^{\ddagger}$ Xiaojuan Liu, ${ }^{*}$ Liang Zhou, ${ }^{\ddagger}$ and Hongjie Zhang*

State Key Laboratory of Rare Earth Resource Utilization, Changchun Institute of Applied Chemistry, Chinese Academy of Sciences, Changchun 130022, People's Republic of China. 


\section{Detailed computational formulas of the energy transfer rate:}

In $\mathrm{Eu}(\mathrm{III})$ complex the organic part has the function of absorbing energy in the UV region and transfer it to the lanthanide ion. The most important transfer pathways that will be considered are between $\mathrm{T}$ and ${ }^{5} \mathrm{D}_{1}, \mathrm{~T}$ and ${ }^{5} \mathrm{D}_{0}$. The energy transfer rate $\mathrm{W}_{\mathrm{ET}}$ between the ligand and metal is given by equation 1 ,

$$
W_{E T}=W_{E T}^{m m}+W_{E T}^{e m}
$$

the first term $W_{E T}^{m m}$ corresponds to the multipolar mechanism and it is given by equation 2 ,

$$
W_{E T}^{m m}=\frac{2 \pi e^{2} S_{L}}{\hbar(2 J+1)} F \sum_{\lambda} \gamma_{\lambda}\left\langle\alpha^{\prime} J^{\prime}\left\|U^{(\lambda)}\right\| \alpha J\right\rangle^{2}+\frac{4 \pi \quad e^{2} S_{L}}{\hbar(2 J+1) G R_{L}^{6}} F \sum_{\lambda} \Omega_{\lambda}^{d e}\left\langle\alpha^{\prime} J^{\prime}\left\|U^{(\lambda)}\right\| \alpha J\right\rangle^{2}
$$

where the $\Omega_{\lambda}^{e d}(\lambda=2,4$, and 6) is the electric dipole contributions to the intensity parameters (with only forced electric dipole). $G$ is the number of components of the initial state of the ligand. $J$ is the total angular momentum quantum number of the Ln ion. $S_{L}$ is the dipole strength associate with the $\phi \rightarrow \phi^{\prime}$ transition in ligands. $\left\langle\alpha^{\prime} J^{\prime}\left\|U^{(\lambda)}\right\| \alpha J\right\rangle$ is the reduced matrix element of the tensor operator $U(l) . \gamma_{\lambda}$ is calculated by equation 3 ,

$$
\gamma_{\lambda}=(\lambda+1) \frac{\left\langle r^{\lambda}\right\rangle^{2}}{R_{L}^{(\lambda+2)}}\left\langle 3\left\|C^{(\lambda)}\right\| 3\right\rangle^{2}\left(1-\sigma_{\lambda}\right)^{2}
$$

where the quantity $\left(1-\sigma_{\lambda}\right)$ is a shielding field due to $5 \mathrm{~s}$ and $5 \mathrm{p}$ filled orbitals of lanthanide ions, which have a radial extension larger than those of the $4 \mathrm{f}$ orbitals. The values of $\sigma_{\lambda}$ are $\sigma_{2}=0.600$, $\sigma_{4}=0.139$ and $\sigma_{6}=0.100 .\left\langle f\left\|C^{(\lambda)}\right\| f\right\rangle$ is a tensor operator of rank $\lambda$ with values $\left\langle 3\left\|C^{(2)}\right\| 3\right\rangle=$ 1.366, $\left\langle 3\left\|C^{(4)}\right\| 3\right\rangle=1.128$, and $\left\langle 3\left\|C^{(6)}\right\| 3\right\rangle=-1.270$ for lanthanide ions. The values of radial integrals $\left\langle r^{\lambda}\right\rangle$ for $\operatorname{Eu(III)~ion~are~}\left\langle r^{2}\right\rangle=0.9175$ a.u., $\left\langle r^{4}\right\rangle=2.0200$ a.u., and $\left\langle r^{6}\right\rangle=9.0390$ a.u., 
respectively.

$F$ corresponds to the temperaturE-dependent factor and contains a sum of Frank Condon factors, which can be calculated by equation 4 ,

$$
F=\frac{1}{\hbar \gamma_{L}} \sqrt{\frac{\ln 2}{\pi}} \exp \left[-\left(\frac{\Delta E}{\hbar \gamma_{L}}\right)^{2} \ln 2\right]
$$

where the factor $\hbar \gamma_{L}$ is the ligand state bandwidth-at-half-maximum and $\Delta E$ is the difference of energy between donor and acceptor state involved in the energy transfer process. In lanthanide complexes, they represent the ligand and Ln ion, respectively.

The second term $W_{E T}^{e m}$ corresponds to the exchange mechanism and it is given by equation 5 .

$$
W_{E T}^{e m}=\frac{8 \pi e^{2}\langle 4 f \mid L\rangle^{4}}{3 \hbar(2 J+1) R_{L}^{4}} F\left\langle\alpha^{\prime} J^{\prime}\|S\| \alpha J\right\rangle^{2} \sum_{m}\left|\left\langle\phi\left|\sum_{k} \mu_{z}(k) s_{m}(k)\right| \phi^{\prime}\right\rangle\right|
$$

where $\langle 4 f \mid L\rangle$ is the radical overlap integral. $s_{m}(=-1,0,1)$ is the spherical component of the spin operator of electron $j$ in the $j$ ligand. $\mu_{z}$ is the component $z$ of its dipole operator. $S$ is the total spin operator of the Ln ion.

The execution of the procedure is included in the LUMPAC program code which is also implemented with the calculation of Judd-Ofelt intensity parameters, radiative and nonradiative emission rates, and quantum yield of the luminescence of Lanthanide complexes. ${ }^{1-7}$

In order to make a comparison between INDO/S and TDDFT, 1-1o and 1-1c are chosen as examples. We have selected B3LYP ${ }^{8-11}$, PBE0 ${ }^{12}$, M062X ${ }^{13-14}$, WB97XD ${ }^{15}$, and B3PW91 ${ }^{16-17}$ as functionals to make TDDFT calculations on their excitation energies in different states. The results have been listed in Table S1 to compare with those of INDO/S method, as shown below. 
Table S1 Comparison of singlet and triplet excitation energies $\left(\mathrm{eV} / \mathrm{cm}^{-1}\right)$ for 1-10 and 1-1c with INDO/S and TD-DFT methods

\begin{tabular}{|c|c|c|c|c|c|c|c|}
\hline & \multirow{2}{*}{ state } & \multirow{2}{*}{ INDO/S } & \multicolumn{5}{|c|}{ TDDFT } \\
\hline & & & B3LYP & PBE0 & M062X & WB97XD & B3PW91 \\
\hline \multirow[t]{3}{*}{$1-10$} & $\mathrm{~T}_{1}$ & $2.37 / 19172$ & $2.57 / 20727$ & $2.53 / 20404$ & $3.04 / 24517$ & $2.69 / 21695$ & $2.54 / 20485$ \\
\hline & $\mathrm{T}_{2}$ & $2.69 / 21670$ & $2.98 / 24034$ & $2.93 / 23630$ & $3.45 / 27842$ & $3.08 / 24840$ & $2.57 / 20727$ \\
\hline & $\mathrm{S}_{1}$ & $3.58 / 28850$ & $3.56 / 28751$ & $3.73 / 30082$ & $4.24 / 34195$ & $4.28 / 34518$ & $3.59 / 28953$ \\
\hline \multirow[t]{2}{*}{$1-1 c$} & $\mathrm{~T}_{1}$ & $1.57 / 12734$ & $1.11 / 8952$ & $1.19 / 9597$ & $1.43 / 11533$ & $1.39 / 11210$ & $0.72 / 5807$ \\
\hline & $\mathrm{S}_{1}$ & $2.87 / 23166$ & $2.35 / 18953$ & $2.45 / 19759$ & $2.68 / 21614$ & $2.31 / 18630$ & $2.04 / 16454$ \\
\hline
\end{tabular}


Table S2 INDO/S-CIS vertical excitation energies(nm) of $\mathrm{S}_{1}$ and $\mathrm{T}_{1}$ state of Eu(acac) ${ }_{3} \mathrm{De}$ complexes, energy transfer, and back transfer $\operatorname{rates}\left(\mathrm{s}^{-1}\right)$

\begin{tabular}{|c|c|c|c|c|c|c|}
\hline & $\mathrm{S}_{1}$ & $\mathrm{~T}_{1}$ & $\mathrm{~T}_{1} \rightarrow{ }^{5} \mathrm{D}_{1}$ & ${ }^{5} \mathrm{D}_{1} \rightarrow \mathrm{T}_{1}$ & $\mathrm{~T}_{1} \rightarrow{ }^{5} \mathrm{D}_{0}$ & ${ }^{5} \mathrm{D}_{0} \rightarrow \mathrm{T}_{1}$ \\
\hline $1-1$ & 342 & 525 & $7.00 \mathrm{E} 3$ & $5.94 \mathrm{E} 3$ & $1.09 \mathrm{E} 4$ & $2.27 \mathrm{E} 0$ \\
\hline $2-1$ & 384 & 541 & 4.33E3 & $5.73 \mathrm{E} 4$ & 7.72E3 & $2.50 \mathrm{E} 1$ \\
\hline $1-2$ & 354 & 522 & $7.65 \mathrm{E} 3$ & $3.91 \mathrm{E} 3$ & $1.17 \mathrm{E} 4$ & $1.46 \mathrm{E} 0$ \\
\hline $2-2$ & 398 & 565 & $2.81 \mathrm{E} 3$ & $1.70 \mathrm{E} 6$ & $6.01 \mathrm{E} 3$ & $8.90 \mathrm{E} 2$ \\
\hline $1-3$ & 355 & 535 & $1.10 \mathrm{E} 4$ & $5.26 \mathrm{E} 4$ & $1.87 \mathrm{E} 4$ & $2.18 \mathrm{E} 1$ \\
\hline $2-3$ & 389 & 559 & $3.80 \mathrm{E} 3$ & 8.37E5 & $7.74 \mathrm{E} 3$ & 4.17E2 \\
\hline $1-4$ & 358 & 518 & $6.87 \mathrm{E} 3$ & $1.81 \mathrm{E} 3$ & $1.02 \mathrm{E} 4$ & $6.55 \mathrm{E}-1$ \\
\hline $2-4$ & 402 & 573 & $2.68 \mathrm{E} 3$ & 4.75E6 & $6.02 \mathrm{E} 3$ & $2.61 \mathrm{E} 3$ \\
\hline $1-5$ & 354 & 520 & $8.23 \mathrm{E} 3$ & $3.27 \mathrm{E} 3$ & $1.24 \mathrm{E} 4$ & $1.21 \mathrm{E} 0$ \\
\hline $2-5$ & 383 & 559 & $1.95 \mathrm{E} 3$ & $5.26 \mathrm{E} 5$ & $4.01 \mathrm{E} 3$ & 2.64E2 \\
\hline $1-6$ & 367 & 522 & $5.86 \mathrm{E} 3$ & $3.20 \mathrm{E} 3$ & 8.97E3 & $1.20 \mathrm{E} 0$ \\
\hline $2-6$ & 417 & 582 & $2.25 \mathrm{E} 3$ & $1.54 \mathrm{E} 7$ & $5.40 \mathrm{E} 3$ & $9.02 \mathrm{E} 3$ \\
\hline $1-7$ & 374 & 526 & $5.15 \mathrm{E} 3$ & $5.80 \mathrm{E} 3$ & $8.16 \mathrm{E} 3$ & 2.25E0 \\
\hline $2-7$ & 434 & 572 & $2.02 \mathrm{E} 3$ & $3.34 \mathrm{E} 6$ & $4.54 \mathrm{E} 3$ & $1.83 \mathrm{E} 3$ \\
\hline $1-8$ & 391 & 552 & $3.35 \mathrm{E} 3$ & $2.15 \mathrm{E} 5$ & $6.44 \mathrm{E} 3$ & $1.01 \mathrm{E} 2$ \\
\hline $2-8$ & 447 & 559 & $2.28 \mathrm{E} 3$ & $5.80 \mathrm{E} 5$ & $4.68 \mathrm{E} 3$ & $2.91 \mathrm{E} 2$ \\
\hline $1-9$ & 410 & 568 & $5.12 \mathrm{E} 3$ & $4.45 \mathrm{E} 6$ & $1.11 \mathrm{E} 4$ & $2.37 \mathrm{E} 3$ \\
\hline $2-9$ & 462 & 579 & $1.80 \mathrm{E} 3$ & $8.47 \mathrm{E} 6$ & $4.25 \mathrm{E} 3$ & 4.87E3 \\
\hline $1-10$ & 436 & 622 & $2.25 \mathrm{E} 3$ & $3.36 \mathrm{E} 9$ & $6.96 \mathrm{E} 3$ & $2.54 \mathrm{E} 6$ \\
\hline $2-10$ & 483 & 583 & $1.49 \mathrm{E} 3$ & $1.24 \mathrm{E} 7$ & $3.61 \mathrm{E} 3$ & $7.36 \mathrm{E} 3$ \\
\hline $1-11$ & 382 & 603 & $9.08 \mathrm{E} 3$ & $1.16 \mathrm{E} 9$ & $2.50 \mathrm{E} 4$ & 7.79E5 \\
\hline $2-11$ & 426 & 603 & $3.59 \mathrm{E} 3$ & 4.34E8 & $9.87 \mathrm{E} 3$ & $2.91 \mathrm{E} 5$ \\
\hline $1-12$ & 368 & 595 & $1.06 \mathrm{E} 5$ & 4.74E9 & $2.79 \mathrm{E} 5$ & $3.03 \mathrm{E} 6$ \\
\hline $2-12$ & 402 & 634 & $3.79 \mathrm{E} 3$ & $2.20 \mathrm{E} 10$ & $1.25 \mathrm{E} 4$ & $1.78 \mathrm{E} 7$ \\
\hline $1-13$ & 320 & 510 & $9.10 \mathrm{E} 3$ & $6.05 \mathrm{E} 2$ & $1.26 \mathrm{E} 4$ & $2.05 \mathrm{E}-1$ \\
\hline $2-13$ & 360 & 554 & $3.16 \mathrm{E} 3$ & $3.52 \mathrm{E} 5$ & $6.24 \mathrm{E} 3$ & $1.69 \mathrm{E} 2$ \\
\hline $1-14$ & 333 & 557 & $4.43 \mathrm{E} 4$ & 8.30E6 & $8.94 \mathrm{E} 4$ & 4.10E3 \\
\hline $2-14$ & 368 & 559 & $5.46 \mathrm{E} 3$ & $1.35 \mathrm{E} 6$ & $1.12 \mathrm{E} 4$ & $6.74 \mathrm{E} 2$ \\
\hline $1-15$ & 339 & 529 & $1.10 \mathrm{E} 4$ & $1.93 \mathrm{E} 4$ & $1.78 \mathrm{E} 4$ & $7.63 \mathrm{E} 0$ \\
\hline $2-15$ & 382 & 554 & $3.32 \mathrm{E} 3$ & $3.77 \mathrm{E} 5$ & $6.55 \mathrm{E} 3$ & $1.82 \mathrm{E} 2$ \\
\hline $1-16$ & 371 & 542 & $7.80 \mathrm{E} 3$ & $1.35 \mathrm{E} 5$ & $1.41 \mathrm{E} 4$ & 5.97E1 \\
\hline $2-16$ & 415 & 580 & $2.49 \mathrm{E} 3$ & $1.24 \mathrm{E} 7$ & $5.88 \mathrm{E} 3$ & 7.17E3 \\
\hline $1-17$ & 344 & 533 & 8.79E3 & $3.25 \mathrm{E} 4$ & $1.47 \mathrm{E} 4$ & $1.33 \mathrm{E} 1$ \\
\hline 2-17 & 392 & 560 & $2.72 \mathrm{E} 3$ & $7.25 \mathrm{E} 5$ & $5.59 \mathrm{E} 3$ & $3.64 \mathrm{E} 2$ \\
\hline
\end{tabular}


Table S3 INDO/S-CIS vertical excitation energies(nm) of $\mathrm{S}_{1}$ and $\mathrm{T}_{2}$ state of Eu(acac) $)_{3} \mathrm{De}$ complex, energy transfer, and back transfer rates $\left(\mathrm{s}^{-1}\right)$

\begin{tabular}{|c|c|c|c|c|c|c|}
\hline & $\mathrm{S}_{1}$ & $\mathrm{~T}_{2}$ & $\mathrm{~T}_{1} \rightarrow{ }^{5} \mathrm{D}_{1}$ & ${ }^{5} \mathrm{D}_{1} \rightarrow \mathrm{T}_{1}$ & $\mathrm{~T}_{1} \rightarrow{ }^{5} \mathrm{D}_{0}$ & ${ }^{5} \mathrm{D}_{0} \rightarrow \mathrm{T}_{1}$ \\
\hline 1-1 & 342 & 453 & $6.62 \mathrm{E} 3$ & $2.02 \mathrm{E}-2$ & $5.71 \mathrm{E} 3$ & $4.26 \mathrm{E}-6$ \\
\hline $2-1$ & 384 & 398 & $3.75 \mathrm{E} 2$ & $7.31 \mathrm{E}-11$ & $1.47 \mathrm{E} 2$ & 7.03E-15 \\
\hline $1-2$ & 354 & 522 & 5.13E3 & $3.58 \mathrm{E}-2$ & $4.60 \mathrm{E} 3$ & $7.85 \mathrm{E}-6$ \\
\hline $2-2$ & 398 & 404 & 3.77E2 & $4.53 \mathrm{E}-10$ & $1.62 \mathrm{E} 2$ & $4.75 \mathrm{E}-14$ \\
\hline $1-3$ & 355 & 474 & $7.58 \mathrm{E} 3$ & $3.49 \mathrm{E}-1$ & $7.44 \mathrm{E} 3$ & $8.37 \mathrm{E}-5$ \\
\hline $2-3$ & 389 & 413 & $7.16 \mathrm{E} 2$ & $1.24 \mathrm{E}-8$ & $3.48 \mathrm{E} 2$ & $1.47 \mathrm{E}-12$ \\
\hline $1-4$ & 358 & 456 & $3.98 \mathrm{E} 3$ & $3.64 \mathrm{E}-3$ & $3.24 \mathrm{E} 3$ & $7.26 \mathrm{E}-7$ \\
\hline $2-4$ & 402 & 393 & - & - & - & - \\
\hline $1-5$ & 354 & 459 & $4.00 \mathrm{E} 3$ & $7.81 \mathrm{E}-3$ & $3.38 \mathrm{E} 3$ & $1.61 \mathrm{E}-6$ \\
\hline $2-5$ & 383 & 394 & $6.20 \mathrm{E} 2$ & $3.48 \mathrm{E}-11$ & $2.30 \mathrm{E} 2$ & $3.16 \mathrm{E}-15$ \\
\hline $1-6$ & 367 & 463 & 3.79E3 & $1.60 \mathrm{E}-2$ & 3.32E3 & $3.43 \mathrm{E}-6$ \\
\hline $2-6$ & 417 & 407 & - & - & - & - \\
\hline $1-7$ & 374 & 415 & $6.97 \mathrm{E} 2$ & $1.71 \mathrm{E}-8$ & $3.44 \mathrm{E} 2$ & $2.07 \mathrm{E}-12$ \\
\hline $2-7$ & 434 & 386 & - & - & - & - \\
\hline $1-8$ & 391 & 385 & $2.06 \mathrm{E} 2$ & $7.88 \mathrm{E}-13$ & $6.72 \mathrm{E} 1$ & $6.29 \mathrm{E}-17$ \\
\hline $2-8$ & 448 & 401 & - & - & - & - \\
\hline $1-9$ & 410 & 438 & $1.71 \mathrm{E} 3$ & $1.98 \mathrm{E}-5$ & $1.13 \mathrm{E} 3$ & $3.20 \mathrm{E}-9$ \\
\hline $2-9$ & 462 & 414 & - & - & - & - \\
\hline $1-10$ & 436 & 396 & $4.20 \mathrm{E} 2$ & $4.85 \mathrm{E}-11$ & $1.61 \mathrm{E} 2$ & $4.55 \mathrm{E}-15$ \\
\hline $2-10$ & 483 & 445 & - & - & - & - \\
\hline $1-11$ & 382 & 488 & $1.13 \mathrm{E} 4$ & 9.99E0 & $1.28 \mathrm{E} 4$ & $2.76 \mathrm{E}-3$ \\
\hline $2-11$ & 426 & 478 & 4.94E3 & $6.08 \mathrm{E}-1$ & $5.08 \mathrm{E} 3$ & $1.53 \mathrm{E}-4$ \\
\hline $1-12$ & 368 & 486 & $6.27 \mathrm{E} 4$ & $3.59 \mathrm{E} 1$ & $6.93 \mathrm{E} 4$ & $9.72 \mathrm{E}-3$ \\
\hline $2-12$ & 433 & 508 & 7.38E3 & 3.32E2 & $1.00 \mathrm{E} 4$ & $1.10 \mathrm{E}-1$ \\
\hline $1-13$ & 320 & 406 & 1.19E3 & $2.57 \mathrm{E}-9$ & $5.23 \mathrm{E} 2$ & $2.77 \mathrm{E}-13$ \\
\hline $2-13$ & 360 & 367 & $4.03 \mathrm{E} 1$ & $3.13 \mathrm{E}-16$ & $9.79 \mathrm{E} 0$ & $1.86 \mathrm{E}-20$ \\
\hline $1-14$ & 333 & 483 & $1.33 \mathrm{E} 4$ & 4.29E0 & $1.43 \mathrm{E} 4$ & $1.13 \mathrm{E}-3$ \\
\hline $2-14$ & 368 & 451 & $3.11 \mathrm{E} 3$ & $8.81 \mathrm{E}-4$ & $2.40 \mathrm{E} 3$ & $1.66 \mathrm{E}-7$ \\
\hline $1-15$ & 339 & 468 & 7.72E3 & $1.16 \mathrm{E}-1$ & $7.18 \mathrm{E} 3$ & $2.64 \mathrm{E}-5$ \\
\hline $2-15$ & 382 & 403 & $4.00 \mathrm{E} 2$ & $4.28 \mathrm{E}-10$ & $1.70 \mathrm{E} 2$ & $4.46 \mathrm{E}-14$ \\
\hline $1-16$ & 371 & 477 & $5.18 \mathrm{E} 3$ & $5.77 \mathrm{E}-1$ & $5.30 \mathrm{E} 3$ & $1.45 \mathrm{E}-4$ \\
\hline $2-16$ & 415 & 418 & $6.54 \mathrm{E} 2$ & $3.77 \mathrm{E}-8$ & $3.37 \mathrm{E} 2$ & 4.74E-12 \\
\hline $1-17$ & 344 & 470 & $6.38 \mathrm{E} 3$ & $1.48 \mathrm{E}-1$ & $6.06 \mathrm{E} 3$ & $3.44 \mathrm{E}-5$ \\
\hline 2-17 & 392 & 407 & $4.11 \mathrm{E} 2$ & $1.42 \mathrm{E}-9$ & $1.85 \mathrm{E} 2$ & $1.57 \mathrm{E}-13$ \\
\hline
\end{tabular}


(a)
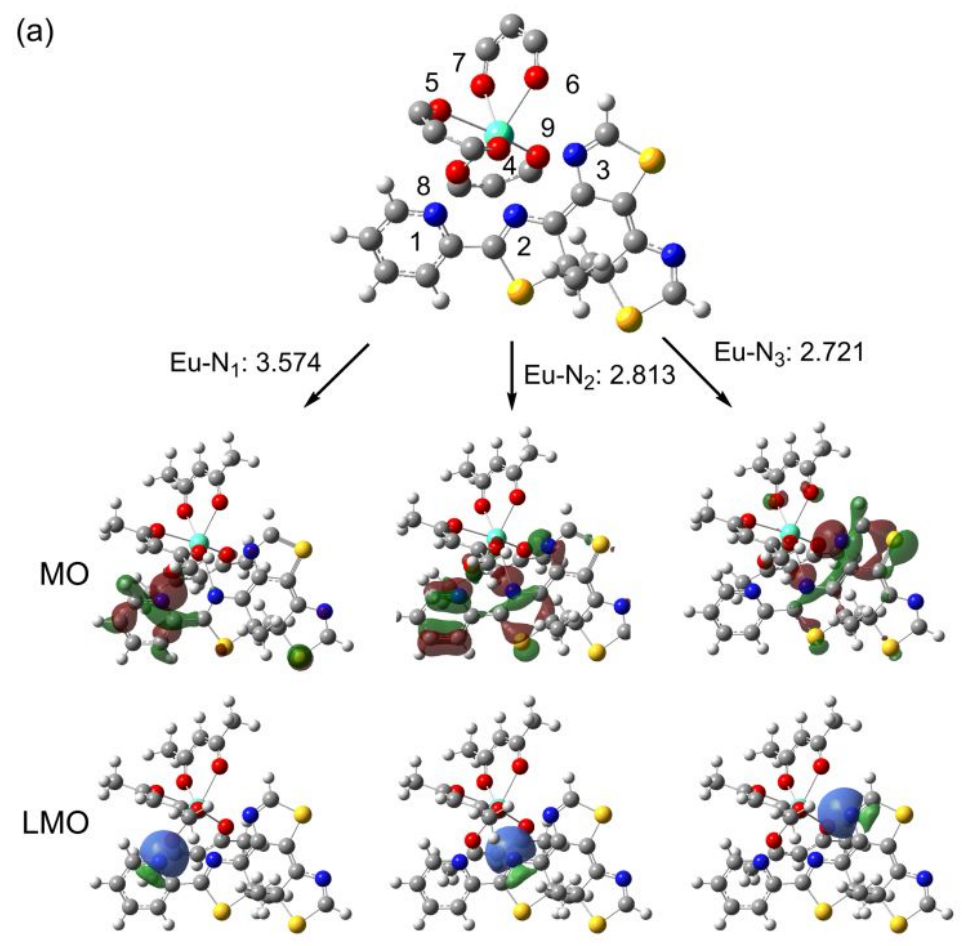

(b)

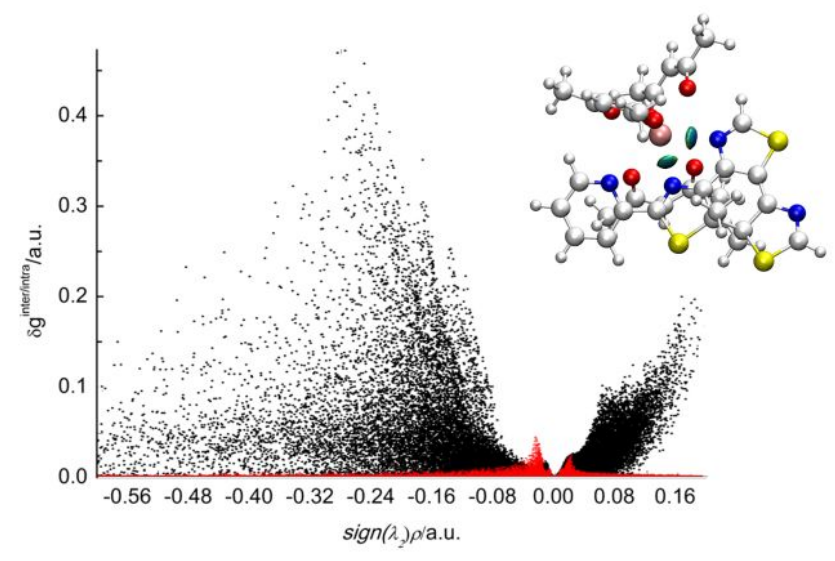

Figure S1. (a) The number of coordinate atoms and spatial distribution of MOs and LMOs contributing to each Eu-N bond in 1-1c. (b) The $\delta g^{\text {inter/intra }}$ vs $\operatorname{sign}\left(\lambda_{2}\right) \rho$ scatter diagrams(red to $\delta g^{\text {inter }}$ and black to $\delta g^{\text {intra }}$, respectively) and spatial isosurface diagram of $\delta g^{\text {inter }}$ (top right) for 1$1 c$ 


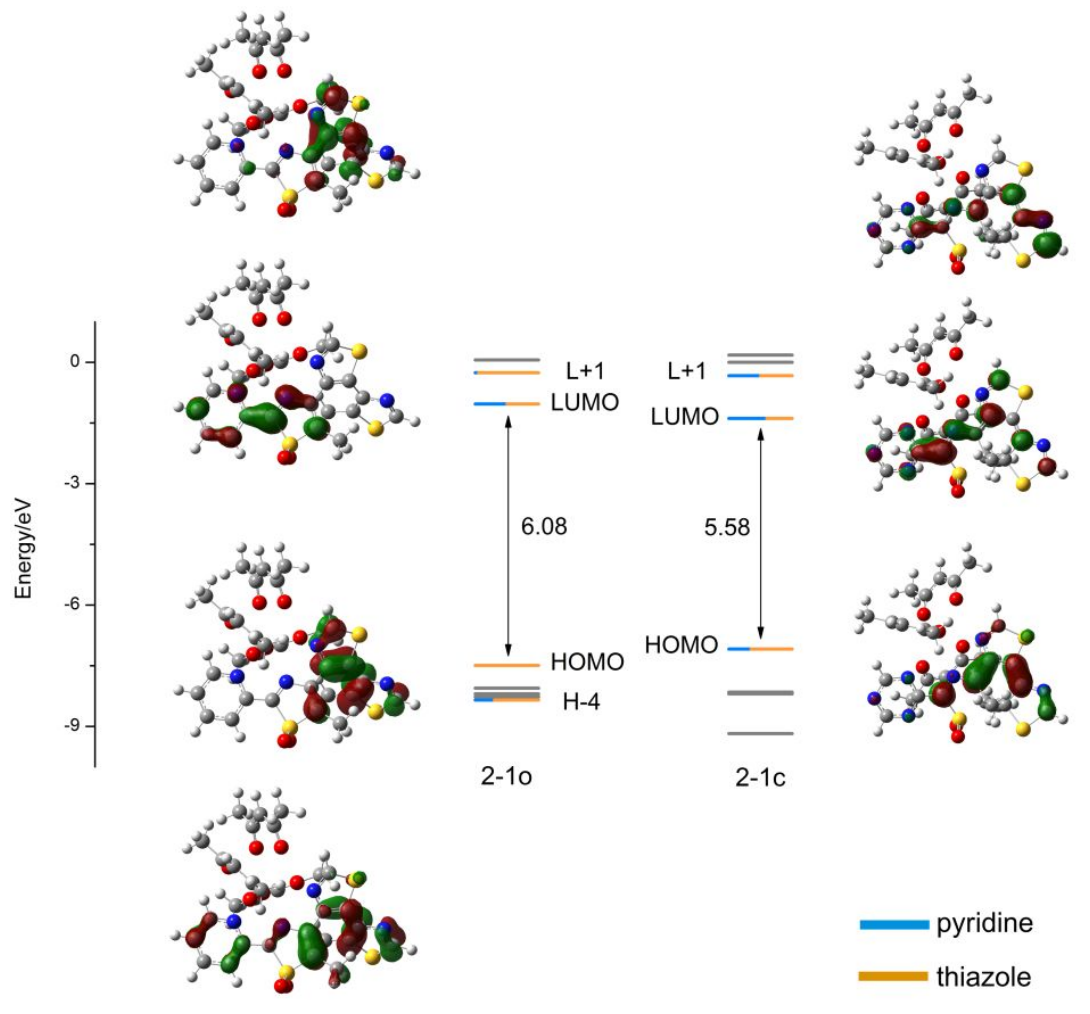

Figure S2. Calculated energy levels, energy gaps (eV), and orbital contributions of molecular orbitals involved in transitions for 2-10 and 2-1c 


\section{Reference}

1. Georgieva, I.; Trendafilova, N.; Zahariev, T.; Danchova, N.; Gutzov, S., Theoretical insight in highly luminescent properties of Eu (III) complex with phenanthroline. J. Lumin. 2018, 202, 192-205.

2. Monteiro, J. H.; de Bettencourt-Dias, A.; Sigoli, F. A., Estimating the donor-acceptor distance to tune the emission efficiency of luminescent lanthanide compounds. Inorg. Chem. 2016, 56 (2), 709-712.

3. Daumann, L. J.; Tatum, D. S.; Andolina, C. M.; Pacold, J. I.; D’Aléo, A.; Law, G.-1.; Xu, J.; Raymond, K. N., Effects of ligand geometry on the photophysical properties of photoluminescent Eu (III) and Sm (III) 1-hydroxypyridin-2-one complexes in aqueous solution. Inorg. Chem. 2015, 55 (1), 114-124.

4. Ilmi, R.; Iftikhar, K., Photophysical properties of Lanthanide (III) 1, 1, 1-trifluoro-2, 4pentanedione complexes with 2, 2' -Bipyridyl: An experimental and theoretical investigation. $J$. Photochem. Photobiol., A 2017, 333, 142-155.

5. $\quad$ Marques, L. F.; Santos, H. P.; D'Oliveira, K. A.; Botezine, N. P.; Freitas, M. C. R.; Freire, R. O.; Dutra, J. D. L.; Martins, J. S.; Legnani, C.; Quirino, W. G., New

photo/electroluminescent europium (III) $\beta$-diketonate complex containing ap, $\mathrm{p}^{\prime}$-disubstituted bipyridine ligand: Synthesis, solid state characterization, theoretical and experimental spectroscopic studies. Inorg. Chim. Acta 2017, 458, 28-38.

6. $\quad$ de Souza Ramos, T. J.; da Silva Viana, R.; Schaidhauer, L.; Cassol, T.; Junior, S. A., Thermoreversible luminescent ionogels with white light emission: an experimental and theoretical approach. J. Mater. Chem. C 2015, 3 (41), 10934-10942.

7. Turchetti, D. A.; Nolasco, M. M.; Szczerbowski, D.; Carlos, L. D.; Akcelrud, L. C., Light emission of a polyfluorene derivative containing complexed europium ions. Phys. Chem. Chem. Phys. 2015, 17 (39), 26238-26248.

8. Becke, A. D., Density-functional exchange-energy approximation with correct asymptotic behavior. Phys Rev A Gen Phys 1988, 38 (6), 3098-3100.

9. Lee C, Y. W., Parr R G., Development of the Colle-Salvetti correlation-energy formula into a functional of the electron density. Phys. Rev. B 1988, 37 (2), 785.

10. Becke, A. D., Density-functional thermochemistry. III. The role of exact exchange. $J$. Chem. Phys. 1993, 98 (7), 5648.

11. Stephens P. J., D. F. J., Chabalowski C. F., Chabalowski, C., Frisch, M. J., Ab initio calculation of vibrational absorption and circular dichroism spectra using density functional force fields. J. Phys. Chem. 1994, 98 (45), 11623-11627.

12. Adamo, C.; Barone, V., Toward reliable density functional methods without adjustable parameters: The PBE0 model. J. Chem. Phys. 1999, 110 (13), 6158-6170.

13. Zhao, Y.; Truhlar, D. G., The M06 suite of density functionals for main group thermochemistry, thermochemical kinetics, noncovalent interactions, excited states, and transition elements: two new functionals and systematic testing of four M06-class functionals and 12 other functionals. Theor. Chem. Acc. 2007, 120 (1-3), 215-241.

14. Zhao, Y.; Truhlar, D. G., Density functionals with broad applicability in chemistry. Accounts. Chem. Res. 2008, 41 (2), 157-167.

15. Chai, J.-D.; Head-Gordon, M., Long-range corrected hybrid density functionals with damped atom-atom dispersion corrections. Phys. Chem. Chem. Phys. 2008, 10 (44), 6615-6620. 
16. Perdew, J. P.; Chevary, J. A.; Vosko, S. H.; Jackson, K. A.; Pederson, M. R.; Singh, D. J.; Fiolhais, C., Atoms, Molecules, Solids, and Surfaces - Applications of the Generalized Gradient Approximation for Exchange and Correlation. Phys. Rev. B 1992, 46 (11), 6671-6687. 17. Perdew, J. P.; Burke, K.; Wang, Y., Generalized gradient approximation for the exchange-correlation hole of a many-electron system. Phys. Rev. B 1996, 54 (23), 16533. 